\title{
Cardiac herniation following intrapericardial pneumonectomy
}

\author{
A. K. D E I R A N I Y A \\ Cardiothoracic Unit, United Birmingham Hospitals, Queen Elizabeth Hospital, \\ Edgbaston, Birmingham 15
}

\begin{abstract}
Deiraniya, A. K. (1974). Thorax, 29, 545-552. Cardiac herniation following intrapericardial pneumonectomy. Cardiac herniation is a rare and catastrophic complication of intrapericardial pneumonectomy. Untreated it is invariably fatal. This paper reports three cases of cardiac herniation following intrapericardial pneumonectomy. In two cases massive haemorrhage complicated the cardiac herniation. All three cases were re-explored with two immediate survivals. The diagnosis, aetiology, haemodynamic effects, and management of this complication are discussed, and previously reported cases are reviewed.
\end{abstract}

Cardiac herniation is a rare, highly lethal complication of intrapericardial pneumonectomy, demanding urgent treatment. The condition presents in the immediate or early postoperative period. Cardiovascular collapse is invariably present. Elevation of the jugular venous pressure and cyanosis in the drainage area of the superior vena cava are frequently noted. Diagnosis rests on an awareness of this condition, its clinical manifestation, and radiological examination.

This paper reports three patients who developed cardiac herniation following intrapericardial pneumonectomy, in two of whom massive haemorrhage complicated the herniation. A tear at the left aspect of the inferior atriocaval junction occurred as a result of cardiac herniation into the right pleural cavity in one case, and in the other the apex of the left ventricle was perforated by a fractured rib following a left pneumonectomy. A survey of the literature has not revealed similar cases. The predisposing factors and methods of prevention and treatment are discussed and previously reported cases are reviewed.

\section{CASE REPORTS}

CASE 1 F. M., a 49-year-old mining inspector, was admitted to hospital on 16 March 1970 following the discovery of a hilar opacity on follow-up examination.

In August 1968 a right nephrectomy was performed for renal carcinoma. His postoperative course was satisfactory. Periodic six-monthly follow-up examinations including chest radiographs were normal until January 1970, when he was referred back with re- trosternal pain and flatulence. Chest radiography revealed an isolated right hilar mass. Tomography showed a well-defined opacity intimately related to the lower lobe bronchus. The appearances were thought to be suggestive of a primary lung neoplasm. Barium meal showed a gastric ulcer.

On admission he was a fit-looking, obese, normotensive man with a well-healed nephrectomy scar. A mass $2 \times 2 \mathrm{~cm}$ was palpable in the right supraclavicular fossa. A chest radiograph on admission showed enlargement of the hilar opacity. Bronchoscopy was normal and histological examination of the excised supraclavicular mass showed a neurolemmoma.

Thoracotomy was performed through the bed of the sixth rib. A lobulated yellowish tumour replacing the hilar lymph nodes and the lymph nodes along the lower and middle lobe bronchi was encountered. Frozen section showed metastatic renal carcinoma. A right intrapericardial pneumonectomy was performed. The pericardial defect measured $6 \times 6 \mathrm{~cm}$. This was not closed. A tube drain was inserted and the chest was closed. Shortly after his return to the ward the patient became restless. Intense cyanosis about the face and neck and hypotension were noted. Ventilation through an endotracheal tube did not materially improve the hypotension or cyanosis. The chest tube which had been inadvertently connected to the suction apparatus, rapidly filled with blood and asystole ensued. The wound was speedily re-opened. A massive haemothorax was evacuated. The heart had herniated through the defect into the right pleural cavity, its apex pointing towards the right paravertebral gutter. Blood was issuing from a $3 \mathrm{~cm}$ tear at the inferior atriocaval junction. After enlarging the defect and repairing the tear, the heart was replaced in the pericardial cavity. Despite rapid transfusion, 
cardiac massage, and ionotropic drugs no sustained cardiac action could be achisved.

Necropsy did not show any further secondary deposit. The heart had been repositioned but it could be dislocated readily. When this was done the superior vena cava and pulmonary artery were twisted and considerable tension was exerted at the medial aspect of the inferior vena cava-right atrial junction where the tear was located (Fig. 1).

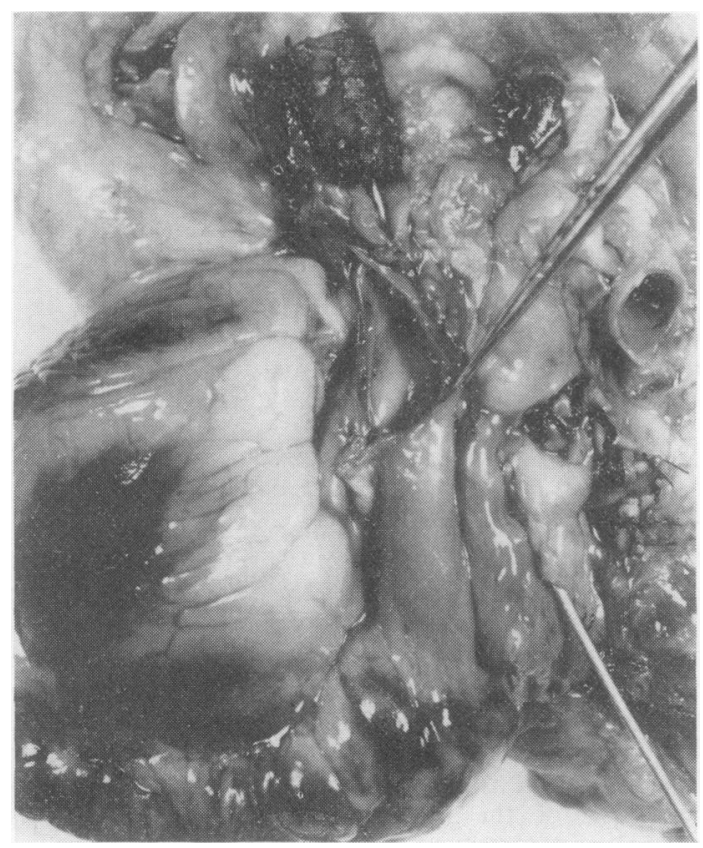

FIG. 1. Postmortem photograph showing the torn aspect of the inferior atriocaval junction. A metal probe can be seen passing from the inferior to the superior vena cava.

CASE 2. V. F., a 71-year-old woman, was admitted to hospital following the discovery of a left upper lobe opacity on a chest film. Physical examination was unremarkable. A chest film showed a large mass arising close to the hilum and extending into the anterior segment of the left upper lobe. Sputum cytology was repeatedly negative. At bronchoscopy no tumour could be seen. Operation was performed through a sixth rib posterolateral incision. A large tumour mass arising from the anterior segment of the left upper lobe and extending centrally to the hilum was found. The subaortic and left tracheobronchial glands were involved. A malignant tissue plaque involved the pleura overlying the aortic course of the recurrent laryngeal and phrenic nerves.

A radical intrapericardial pneumonectomy with deliberate division of the vagus and phrenic nerves was carried out. The resulting defect measured $7.5 \times 7.5 \mathrm{~cm}$. No attempt was made to close the defect. A pleural tube was inserted and the chest高 wound was closed. An immediate postoperative chest흠 film showed elevation of the left leaf of the diaphragm $\frac{\bar{S}}{\partial}$ (Fig. 2).

Sixteen hours postoperatively the patient became restless with profound hypotension and bradycardia. The jugular veins were engorged. An unusually vigorous $\overrightarrow{0}$ pulsation was easily visible in the left second inter- $-\overrightarrow{-}$ costal space in the mid clavicular line. An electro- $\vec{\omega}$ cardiogram showed ischaemic ST segment changes and complete heart block. A tentative diagnosis of cardiac herniation was made. A chest film showed anî elevated diaphragm and dislocation of the heart into 6 the left pleural cavity (Fig. 3).

An isoprenaline drip was started and the patient $\frac{A}{G}$ was returned to the operating theatre. On re-openingo the chest it was apparent that the heart had herniated through the pericardial defect. The margins of the $\vec{\omega}$ defect were firmly encircling the ventricle caudal to the atrioventricular groove. The constricting ring was $\frac{\mathbb{D}}{\mathbb{D}}$ divided and the herniated heart was repositioned. A Dacron sheet was sutured with interrupted sutures to the edges of the defect. Isoprenaline support was required for 24 hours following re-exploration. Follow- $\vec{\theta}$ ing discontinuation of the isoprenaline she developed $\square$ nodal rhythm, which reverted to sinus rhythm after $\square$ the administration of $0.6 \mathrm{mg}$ atropine sulphate intra-응 venously. She was discharged home on the fourteenth postoperative day.

CASE 3 G. P.. a 65-year-old retired farm worker. presented with persistent cough and haemoptysis. $\overrightarrow{\overrightarrow{0}}$ Clinical examination was unremarkable. A chest 3 radiograph revealed an opacity in the apical segment of the left lower lobe. Bronchoscopy was normal.

At operation an advanced tumour was found which had invaded the chest wall and the lung hilum. Ano intrapericardial left pneumonectomy was performed®ֶ in which most of the pericardium overlying the left ventricle was excised together with segments of the 3 fifth and sixth ribs. The resultant defect was not: repaired. The seventh rib was fractured obliquely in윽 the mid-axillary line by the self-retaining retractor.

Two hours after leaving theatre the patient became응 pale and hypotensive. The left hemithorax was dull to percussion, but as an intercostal drain had not been음 inserted there was no confirmatory evidence of massive intrapleural haemorrhage. A clinical diagnosis of slipped vascular ligature was made and the patientr returned to theatre immediately. On re-opening theN left hemithorax a massive haemothorax of approxi- $\omega$ mately 2 litres was found and evacuated. The heart had dislocated into the left chest and the apex ofo the left ventricle was impaled on the sharp end of the fractured seventh rib. The heart was replaced in the ${ }^{+}$ pericardium and the ventricular tear was closed with interrupted sutures. The pericardial defect was bridgedo with a sheet of woven Dacron cloth and the chest疋 was closed.

His cardiovascular status gave rise to little anxiety in the postoperative period, but the combination of 


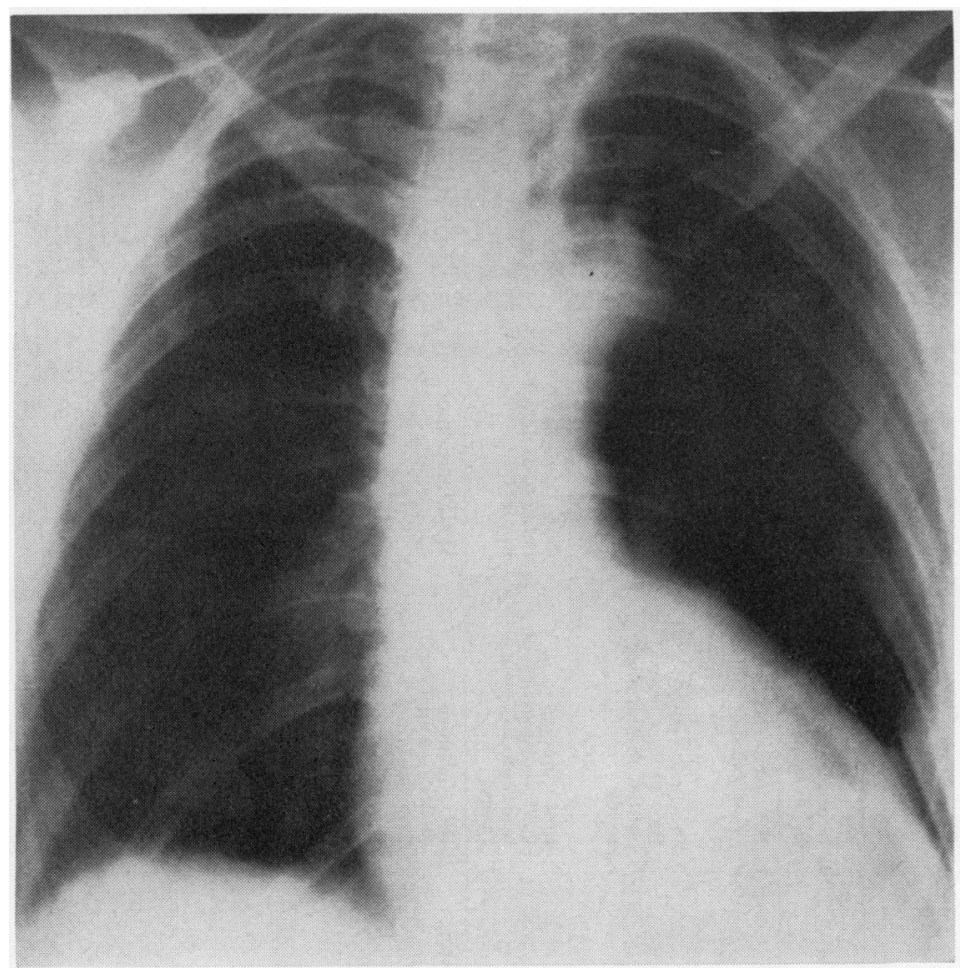

FIG. 2. Immediate postoperative chest film-note elevation of left leaf of diaphragm.

FIG. 3. Chest film 17 hours postoperatively. Note the marked left shift of the cardiac shadow and the central tracheal air shadow. The upper border of the herniated heart can be seen in the left hemithorax

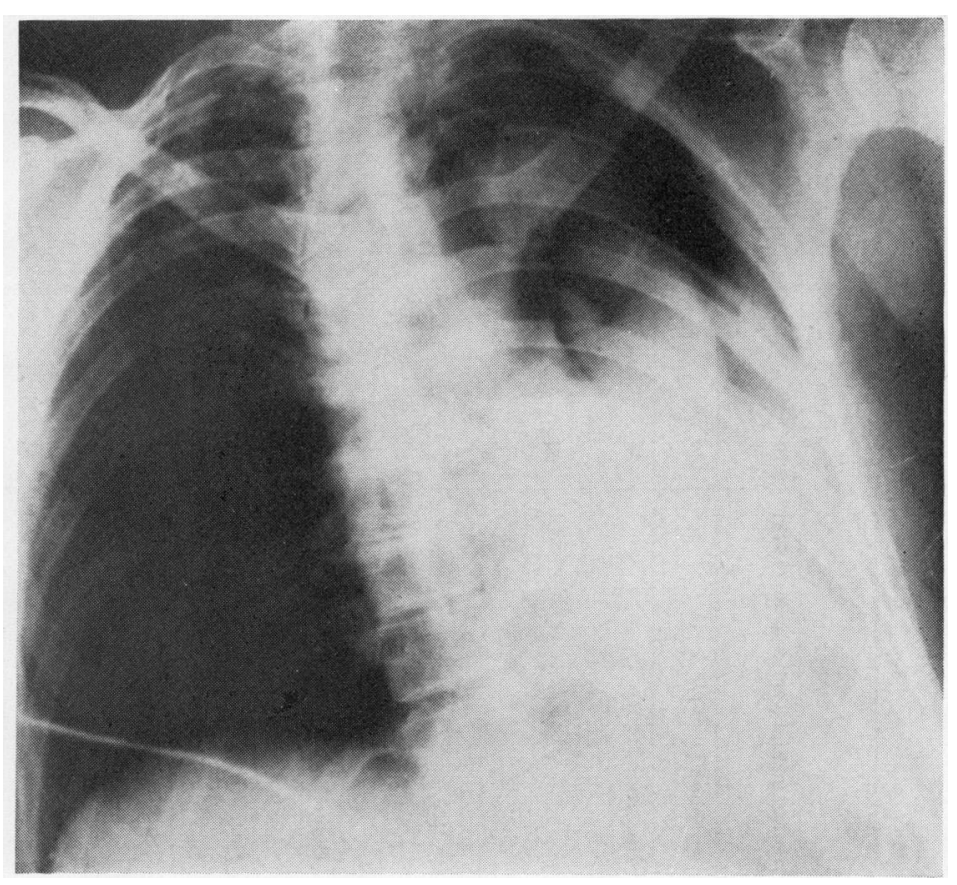




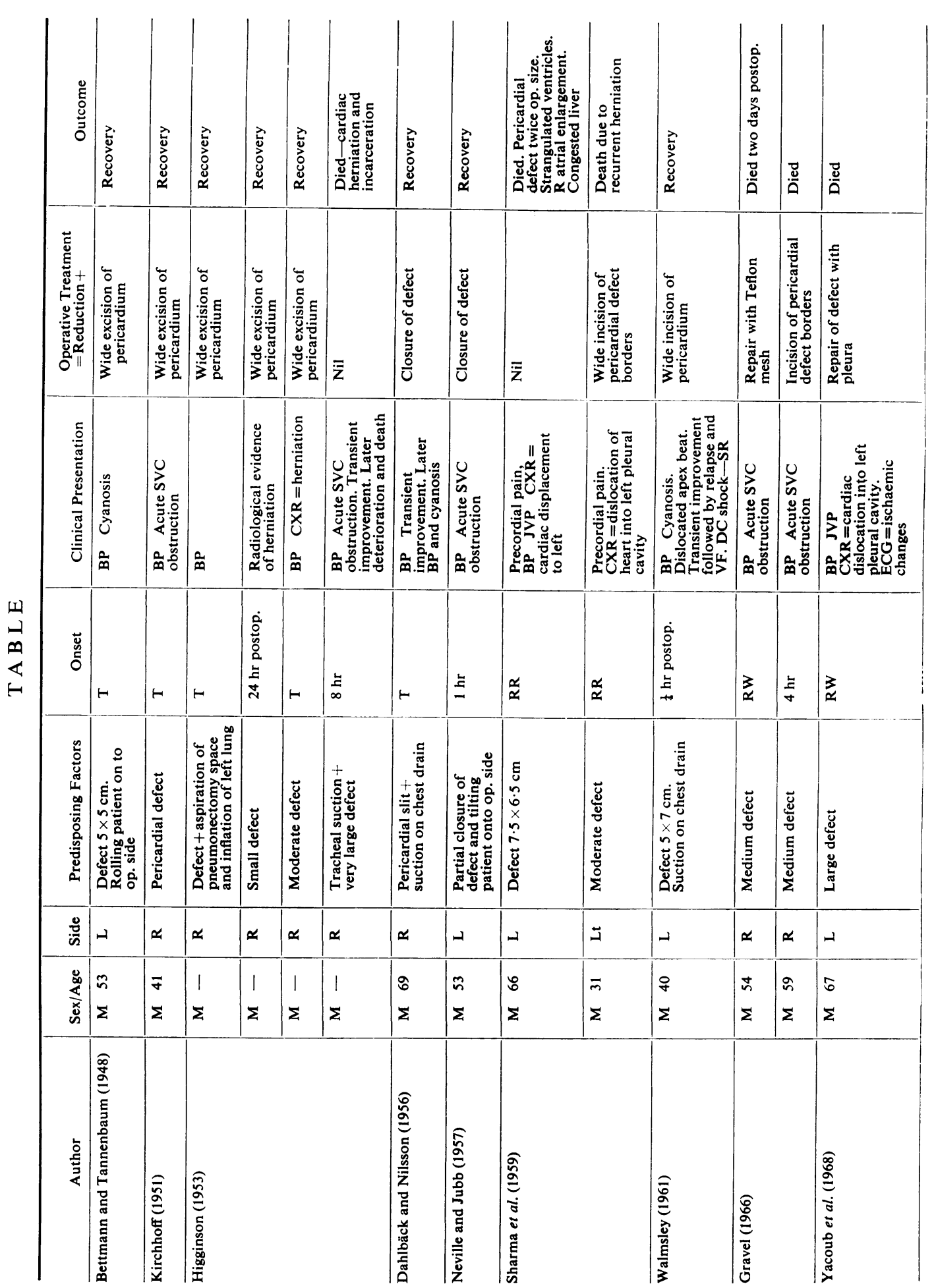

里

항

$\overrightarrow{0}$

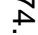

잉

흥

\%ัญ

윽

궁

항

离

훅

ㅇํำ

음

을

ก

N

ర్

응

$\stackrel{\mathscr{Q}}{\longrightarrow}$

꿍

웅

吕

뭉 

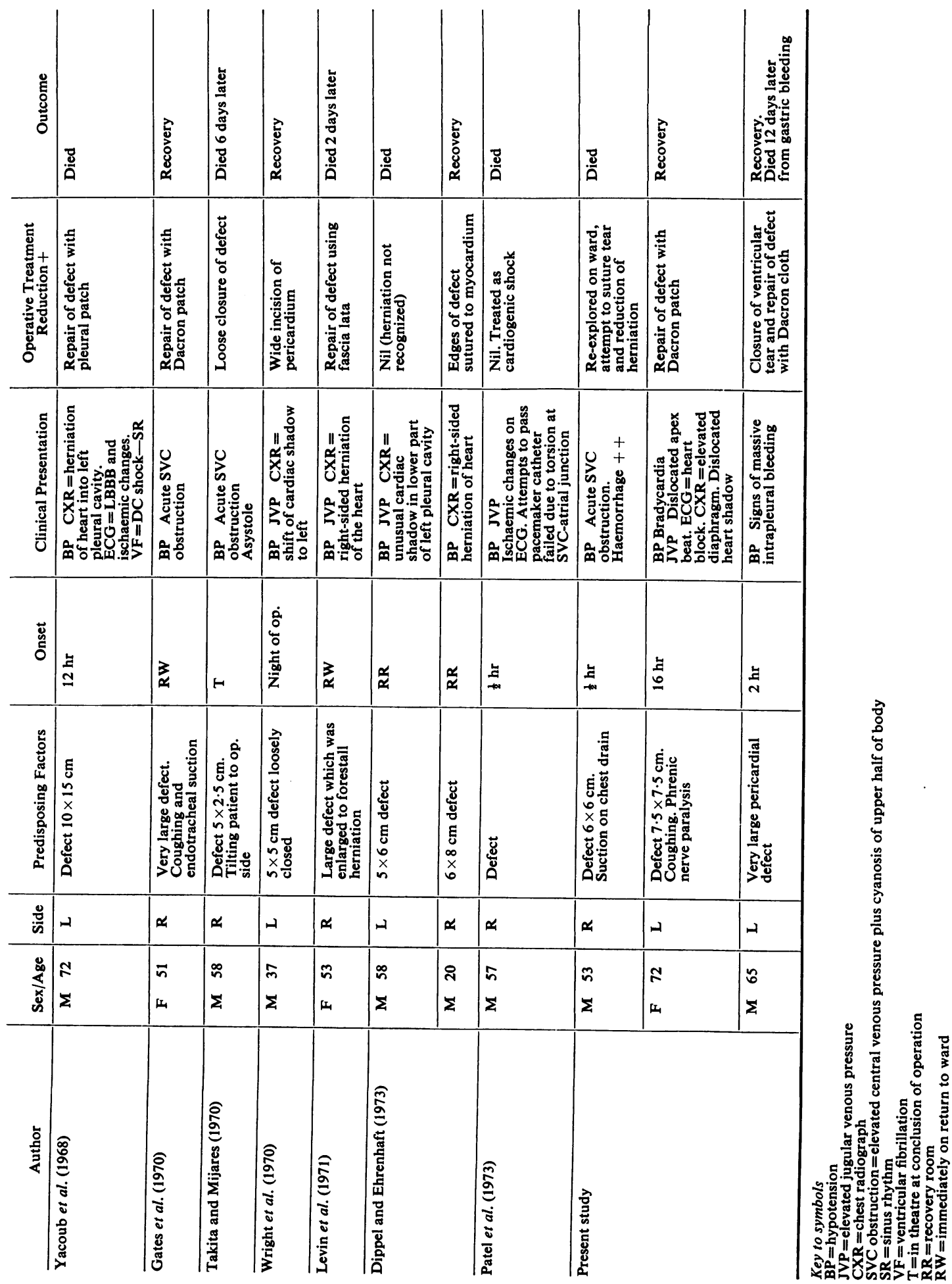
poor respiratory function and paradoxical movement of the left chest wall produced marked dyspnoea. One week after surgery he developed bronchopneumonia which responded to antibiotic therapy. A massive haematemesis from a duodenal ulcer led to his sudden death on the twelfth postoperative day.

\section{DISCUSSION}

Intrapericardial ligation of the pulmonary vessels has allowed a safer and more radical excision of lung tumours encroaching on the hilum or actually invading the pericardium (Allison, 1946). The resultant pericardial defect, if not repaired, however, exposes the patient to the risk of cardiac herniation into the pneumonectomy space.

Although most documented instances of cardiac herniation have occurred through surgically created defects, fatal herniation of the heart through a congenital defect, situated high up on the left side of the pericardial sac, has been reported on three occasions (Boxall, 1886; Sunderland and Wright-Smith, 1944; Bruning, 1962). Cardiac herniation through pericardial tears has resulted from severe blunt injury to the chest (Warburg, 1938; Beck, 1947; Wright, Nelson, Johnson, and McMillan, 1970). I have encountered this complication of blunt thoracic trauma in two patients, both of whom died of associated injuries. The heart prolapsed into the left pleural cavity through a longitudinal tear in the pericardium, which extended from the pericardial reflection onto the pulmonary artery to the diaphragm.

Bettmann and Tannenbaum (1948) reported the first case of cardiac herniation following an intrapericardial pneumonectomy. Since then at least 24 further cases, including the three presented in this paper, have appeared in the English literature (Kirchhoff, 1951; Higginson, 1953; Dahlbäck and Nilsson, 1956; Neville and Jubb, 1957; Sharma, Bates and Hurt, 1959; Walmsley, 1961; Gravel, 1966; Yacoub, Williams and Ahmad, 1968; Gates, Sette, and Cope, 1970; Takita and Mijares, 1970; Wright et al., 1970; Levin, Penfield-Faber, and Carlton, 1971; Dippel and Ehrenhaft, 1973; Patel, Shrivastav, and Sabety, 1973). Undoubtedly further unreported and unsuspected cases must have occurred. Of the 25 cases, three were females, a reflection of the prevalence of lung cancer in males. There were 14 right-sided and 11 left-sided herniations of the heart. Seven of the former and five of the latter died, giving an overall mortality of $50 \%$. In four cases the diagnosis was missed. All four died. Of 21 patients treated surgically, 12 survived.

The condition may present at the conclusion of the operation or in the very early postoperative period (Higginson, 1953; Dahlbäck and Nilsson, $\frac{\bar{C}}{\frac{\sigma}{\sigma}}$ 1956; Sharma et al., 1959). All cases occurred $\frac{\bar{\sigma}}{\omega}$ within the first 24 hours. The onset is dramatic, $\underset{\mathbb{}}{\overparen{ }}$ with sudden profound hypotension and tachycardia. Cyanosis in the drainage area of the superior vena cava, most marked around the face and $\vec{\circ}$ neck, and elevation of the jugular venous pressure $\overrightarrow{-}$ are frequently noted, particularly in right-sided $\vec{\omega}$ herniation of the heart (Kirchhoff, 1951; Gravel, 1966; Gates et al., 1970; Takita and Mijares, 1970). $\times$ There is no clinical evidence of respiratory obstruction, contralateral pneumothorax or trachealior shift. Oxygen administration has no effect on the cyanosis which is due to obstruction of the ${ }_{\circ}^{\mathrm{r}}$ superior vena cava. An unduly prominent apical thrust is present in some cases of left-sided hernia- $\vec{\omega}$ tion of the heart (Walmsley, 1961). The expected absence of cardiac impulse in the left hemithorax $\underset{\mathbb{D}}{\stackrel{+}{*}}$ in cases of right-sided herniation of the heart has, $\frac{3}{8}$ surprisingly, not been documented. Transient只 clinical improvement after the onset of herniation, $\vec{\varphi}$ as evidenced by sudden hypotension, occurs in some cases. All, however, relapsed into profound shock within a short time (Higginson, 1953; Dahlbäck and Nilsson, 1956; Yacoub et al., 1968). These warning episodes should not be ignored. Radiological examination with critical appraisalo of the chest film is essential.

The radiological appearance of right-sided $\overrightarrow{\vec{\sigma}}$ cardiac herniation is unmistakable. Left-sided 3 herniation of the heart is less easy to recognize. A rounded opacity in the lower part of the left. hemithorax caused by the strangulated ventricular mass is visible in addition to the left shift of the cardiac shadow. There may be a constriction: between the cardiac mass and the great vessels, $\frac{}{3}$ the heart assuming a spherical shape. The apex may contact the lateral chest wall or be posteriorly directed into the posterior costophrenic sulcus (Gates et al., 1970).

The effects of cardiac herniation are due to combination of cardiac malposition with subse quent torsion of the great vessels, obstruction tof the outflow tracts of the heart, and strangulation of the prolapsed ventricles by the borders of the pericardial defect. The haemodynamic distur- $-\sigma$ bance in right-sided herniation is more related to cardiac malposition. The anticlockwise rotation of the dextroposed heart results in a gross degrees of torsion of the atriocaval junctions and ventri $=$ cular outflow tract obstruction. We believe that the shearing stress at the medial aspect of theD inferior atriocaval junction, resulting from for $\frac{\text { के }}{\mathrm{D}}$ cible dislocation of the heart into the right pleuraf cavity when suction was applied to the chest tuber 
was responsible for the tear at the inferior atriocaval junction in our first patient. It is possible that trauma to that particular area by the inferior border of the defect may have contributed to the development of the tear.

The effects of left-sided herniation on the circulation result more from compression of the ventricular muscle by the edges of the pericardial defect. Increasing oedema and congestion of the herniated heart caused by the constricting edges of the defect aggravates the condition. The damage to the myocardium soon becomes irreversible. Electrocardiographic ischaemic changes characterized by ST segment changes, widened complexes, and disorders of conduction have been documented (Yacoub et al., 1968; Patel et al., 1973) and occasionally led to misdiagnosis (Patel et al., 1973). Ventricular fibrillation when it occurs may respond to DC shock (Walmsley, 1961; Yacoub et al., 1968). Prompt relief of the herniation after restoration of heart action has resulted in survival (Walmsley, 1961).

The presence of a medium or large defect in a pericardial cavity free of adhesions is a prerequisite for cardiac herniation to occur. Defects measuring $5 \times 5 \mathrm{~cm}$ or more are the most dangerous, although herniation has on occasions occurred through small defects (Neville and Jubb, 1957; Takita and Mijares, 1970). The absence of any recorded cases of late herniation is probably the result of rapid development of adhesions between the heart and the parietal pericardium. Allison (1946) has shown that the edges of the defect became adherent to the heart within three days.

Predisposing factors have included the application of suction to chest tubes (Walmsley, 1961; Dahlbäck and Nilsson, 1956), positive pressure inflation of the remaining lung while aspirating air from the pneumonectomy space (Higginson, 1953), tracheal aspiration and coughing (Gates $e t$ al., 1970) or changes in the position of a patient during transport from the operating theatre to the ward (Neville and Jubb, 1957; Takita and Mijares, 1970).

Prophylactic measures against possible herniation of the heart should be taken in all cases where a pericardial defect has been created. Wide excision of the pericardium is certainly no insurance against cardiac herniation and incarceration (Higginson, 1953; Levin et al., 1971; Yacoub et al., 1968). Very small defects are probably harmless and can be closed with direct suture. It is our belief that all medium and large defects must be repaired. Strong pleura, if available, can be used to close the defect but the use of thin pleura is not reliable. Tubbs (1959) suggested closure of the defect by a latticework of catgut.

We have found the use of pericardial slings fashioned from the edges of the defect and swung across it with fixation to a point diametrically opposite the origin of the sling effective in medium-sized defects. More than one sling can be fashioned so that only small insignificant gaps remain in the pericardium. The use of synthetic material to repair the defect is open to the theoretical objection that it may abrade the surface of the heart (Levin et al., 1971). We have used Dacron on many occasions prophylactically and twice in the treatment of cardiac herniation with satisfactory results. However, its presence may perpetuate any sepsis should the pneumonectomy space become infected.

The use of fascia lata to repair the defect has been advocated by Levin et al., (1971). A simple technique involves the suturing of the edges of the defect to the myocardium at $1 \mathrm{~cm}$ intervals, avoiding the coronary vessels (Dippel and Ehrenhaft, 1973). In the treatment of the established condition urgent operative intervention is essential because damage to the myocardium soon becomes irreversible. Ionotropic drug support may be required preoperatively and after the relief of the herniation.

The reduction of the herniated heart is effected by incising the constricting borders of the defect.

Measures to prevent recurrence of cardiac herniation should also be carried out. We do not favour the use of fascia lata or the method advocated by Dippel and Ehrenhaft (1973). Our objection to the use of fascia lata is based on the fact that it is inconvenient to obtain in the course of the operation, and that it inflicts unnecessary trauma on the patient. We have doubts about the security of anchorage achieved by suturing the myocardium to the edges of the pericardial defect as suggested by Dippel and Ehrenhaft (1973).

Simple incision of the border of the defect and reduction of the heart is not adequate treatment, for fatal recurrent herniation has followed its use (Sharma et al., 1959). We do not believe that enlarging the defect by wide excision in either right or left-sided herniation is a guarantee against further recurrence.

I should like to thank Mr. A. G. Norman and Mr. D. B. Clarke, under whose care the patients were admitted, and Mr. H. C. Nohl-Oser for their help and advice. 


\section{REFERENCES}

Allison, P. R. (1946). Intrapericardial approach to the lung root in the treatment of bronchial carcinoma by dissection pneumonectomy. Journal of Thoracic Surgery, 15, 99.

Beck, C. S. (1947). Pressures on the heart. Southern Surgeon, 13, 348.

Bettmann, R. B. and Tannenbaum, W. J. (1948). Herniation of the heart through a pericardial incision. Annals of Surgery, 128, 1012.

Boxall, R. (1886). Incomplete pericardial sac; escape of heart into the left pleural cavity. Transactions of the Obstetrical Society, 28, 209.

Bruning, E. G. H. (1962). Congenital defect of the pericardium. Journal of Clinical Pathology, 15, 133.

Dahlbäck, O. and Nilsson, E. (1956). Incarceration of the heart following right pneumonectomy. Acta Chirurgica Scandinavica, 110, 447.

Dippel, W. F. and Ehrenhaft, J. L. (1973). Herniation of the heart after pneumonectomy. Journal of Thoracic and Cardiovascular Surgery, 65, 207.

Gates, G. F., Sette, R. S., and Cope, J. A. (1970). Acute cardiac herniation with incarceration following pneumonectomy. Radiology, 94, 561.

Gravel, J. A. (1966). Herniation of the heart, a hazard of thoracic surgery. Report of two fatal cases. Canadian Journal of Surgery, 9, 72.

Higginson, J. F. (1953). Block dissection in pneumonectomy for carcinoma. Journal of Thoracic Surgery, 25, 582.

Kirchhoff, A. C. (1951). Herniation of the heart. Report of a case. Anaesthesiology, 12, 774.

Levin, P. D., Penfield-Faber, L., and Carlton, R. A. (1971). Cardiac herniation after pneumonectomy. Journal of Thoracic and Cardiovascular Surgery, 61, 104.
Neville, W. E. and Jubb, E. D. (1957). SuccessfuE treatment of cardiac herniation through $2 \overline{\bar{c}}$ surgical rent in the pericardium. Americar Journal of Surgery, 93, 1038.

Patel, D. R., Shrivastav, R., and Sabety, A. M. (1973)心 Cardiac torsion following intrapericardial pneumonectomy. Journal of Thoracic and Cardio? vascular Surgery, 65, 626.

Sharma, V. N., Bates, M., and Hurt, R. L. (1959)ळ Herniation of the heart after intrapericardial pneumonectomy for bronchial carcinoma? Thorax, 14, 36.

Sunderland, S. and Wright-Smith, R. J. (1944). Con'r genital pericardial defects. British Heart Journal 6, 167.

Takita, H. and Mijares, W. S. (1970). Herniation of the heart following intrapericardial pneumonectomy. Report of a case and review. Journal ots

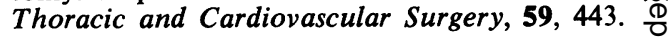

Tubbs, O. S. (1959). As quoted by Sharma et al: Thorax, 14, 36.

Walmsley, D. A. (1961). Herniation of the heart after intrapericardial pneumonectomy. Lancet, $1 \overrightarrow{\vec{b}}$ 645.

Warburg, R. (1938). Subacute and Chronic Peri cardial and Myocardial Lesions Due to Nor Penetrating Traumatic Injuries. Oxford Univer sity Press, London.

Wright, M. P., Nelson, C., Johnson, A. M., anषు McMillan, I. K. R. (1970). Herniation of theD heart. Thorax, 25, 656.

Yacoub, M. H., Williams, W. G., and Ahmad, A옴 (1968). Strangulation of the heart following intrapericardial pneumonectomy. Thorax, 23? 261.

Requests for reprints to: A. K. Deiraniya, F.R.C.S.Q़ Cardiothoracic Unit, United Birmingham Hospitals Queen Elizabeth Hospital, Edgbaston, Birmingham, $15_{\overline{0}}^{x}$ 